\title{
HABILITACIÓN DE LOS MODOS ACTIVOS DEL LENGUAJE A PARTIR DEL MODO REACTIVO OBSERVAR ${ }^{a}$
}

\author{
Agustín Daniel Gómez Fuentes*, Enrique Zepeta García, Zaira Jacqueline García Pérez y Cecilia Magdalena Molina López \\ Universidad Veracruzana
}

Recibido, octubre 11/2014

Concepto de evaluación, noviembre 28/2014

Aceptado, diciembre 30/2014
Referencia: Gómez Fuentes, A.D., Zepeta García, E., García Pérez, Z.J., \& Molina López, C.M. (2015). Habilitación de los modos activos del lenguaje a partir del modo reactivo observar. Acta Colombiana de Psicología, 18(1), 13-24. DOI: 10.14718/ACP.2015.18.1.2

Resumen

El propósito del estudio fue evaluar el efecto de habilitación lingüística en los modos activos del lenguaje señalar, hablar y escribir a partir del modo reactivo observar. Participaron 15 estudiantes experimentalmente ingenuos, doce hombres y tres mujeres, de 14 años de edad, alumnos de tercer grado de una escuela secundaria pública de la ciudad de Xalapa. Se utilizó un arreglo experimental de igualación de la muestra de segundo orden con figuras geométricas y un diseño experimental de tres grupos con prueba inicial, fase de familiarización, fase de exposición y prueba de habilitación. Los resultados sugieren que la exposición al modo observar habilitó las respuestas de igualación en los tres modos activos del lenguaje con distintos niveles de ejecución. El máximo nivel se obtuvo en hablar, el segundo en escribir y el mínimo en señalar. El porcentaje de respuestas de discriminación fue un poco más alto en semejanza en color, seguido por diferencia en color y forma, y un poco más bajo en semejanza en forma. Los resultados se discuten con base en el concepto de habilitación lingüística.

Palabras clave: Lenguaje, conducta humana, lenguaje hablado

\section{ENABLING ACTIVE MODES OF LANGUAGE FROM THE REACTIVE OBSERVING MODE}

\begin{abstract}
The purpose of the study was to assess the effect of enabling linguistic active modes of language by pointing out, writing and speaking from the reactive mode observing. Participants were 15 experimentally naive students, twelve men and three women, aged 14, who attended third grade in a public Junior High School in the city of Xalapa. A second-order matching to sample task with geometric figures was used. The design chosen was experimental with three groups and consisted of an initial test, familiarization phase, exposure phase and final test. The results suggest that the exposure to the observing mode enabled the matching responses in the three active modes of language with different performance levels. The largest level was speaking, then was writing and the lower was pointing out. The percentage of response discrimination was slightly higher in color similarity, followed by the difference in color and form and slightly lower percentage in shape similarity. The results are discussed based on the concept of enabling linguistic modes.

Key words: Language, human behavior, spoken language
\end{abstract}

* Instituto de Psicología y Educación, Universidad Veracruzana. Agustín Melgar y Juan Escutia s/n, Colonia Revolución, 91300 Xalapa, Veracruz.Teléfono:(52)228-8158619.d1031443576@aol.com,enriquezepeta@hotmail.com,nefertary2109@hotmail.com,cemolina@uv.mx a Los autores agradecen la participación de José Miguel Muñoz Matías, alumno de la Maestría en Investigación en Psicología Aplicada a la Educación. 


\title{
HABILITAÇÄO DOS MODOS ATIVOS DA LINGUAGEM A PARTIR DO MODO REATIVO OBSERVAR
}

\author{
Resumo
}

\begin{abstract}
O propósito do estudo foi avaliar o efeito de habilitação linguística nos modos ativos da linguagem assinalar, falar e escrever a partir do modo reativo observar. Participaram 15 estudantes experimentalmente ingênuos, doze homens e três mulheres, de 14 anos de idade, alunos de uma escola secundária pública da cidade de Xalapa. Utilizou-se um arranjo experimental de igualação da mostra de segunda ordem com figuras geométricas e um desenho experimental de três grupos com teste inicial, fase de familiarização, fase de exposição e teste de habilitação. Os resultados sugerem que a exposição ao modo observar habilitou as respostas de igualação nos três modos ativos da linguagem com diferentes níveis de execução. O nível máximo foi obtido em falar, o segundo em escrever e o mínimo em assinalar. A porcentagem de respostas de discriminação foi um pouco mais alta em semelhança em cor, seguido por diferença em cor e forma, e um pouco mais em semelhança em forma. Os resultados se discutem com base no conceito de habilitação linguística.

Palavras chave: Linguagem, conduta humana, linguagem falada.
\end{abstract}

\section{INTRODUCCIÓN}

El diseño, aplicación y evaluación de los planes y programas de estudio de la educación básica se sustenta en supuestos que mantienen la lógica tradicional centrada en el que enseña. Esta enfatiza que la demostración clara, lógica y coherente de los contenidos y prácticas de conocimiento conduce a una comprensión equivalente en el aprendiz (Ribes, 2008). La comprensión ha sido estudiada tradicionalmente como un proceso cognitivo asociado directa o indirectamente al lenguaje. Los modelos propuestos enfatizan procesos de índole mental, internos al organismo que interactúan con representaciones de los eventos experimentados, almacenados en la memoria (Ribes, Ibáñez \& Pérez Almonacid, 2014).

El diseño de los planes y programas de estudio de la educación básica se organiza a partir de campos formativos y dominios de conocimiento: científico, artístico, religioso y tecnológico (Secretaria de Educación Básica [SEP], 2011a). El término competencia concebido como capacidad general y saberes móviles asumidos en la Reforma Integral de la Educación Básica (RIEB) se fundamenta en diferentes enfoques teóricos sustentados en concepciones paradigmáticas distintas (SEP, 2011b). La operación de los programas y planes de estudio a partir del Acuerdo 592, con base en competencias generales, es cuestionable, pues en la enseñanza y el aprendizaje de los dominios del conocimiento no se identifican competencias específicas que orienten y evalúen su operación (SEP, 2011c); además, el trayecto formativo desde preescolar a secundaria carece de una articulación adecuada entre los distintos niveles. Sin embargo, señala Ribes $(2006,2011)$ que el término competencia puede constituirse en un instrumento útil desde un cuerpo teórico sustentado en la ciencia del comportamiento para precisar los requerimientos de formación académica y práctica de los estudiantes.

Los elementos o factores que debe contemplar la planeación del proceso educativo centrado en el aprendiz pueden estructurase con base en tres ejes, que en cada nivel atienden a dimensiones diferentes del proceso educativo (Ribes, 2008). En el primer eje, se incluyen los dominios del conocimiento, conjuntamente con los aspectos psicológicos que deben ser considerados en el aprendizaje: los niveles de aptitud funcional y habilidades que constituyen las competencias, así como los modos del lenguaje involucrados. En el segundo, los dominios que delimitan las competencias de vida, con criterios funcionales y habilidades derivados de circunstancias y problemas de la vida cotidiana (i.e., salud). En el tercero, los criterios y modos de conocimiento posibles (i.e., ordinario, científico, etc.), así como los modos de vida significativos, y sus criterios (i.e., individual, interpersonal, etc.).

La conjunción de estos factores, de acuerdo con el autor citado determina la circunstancia didáctica pertinente como criterio de enseñanza para cada una de las intersecciones posibles. A pesar de que el proceso educativo es un todo integrado, para efectos de análisis, el presente estudio estará vinculado únicamente con los modos del lenguaje involucrados en el dominio de los contenidos del conocimiento, primer eje de la propuesta.

El dominio de los contenidos del conocimiento se relaciona, primero, con las interacciones lingüísticas que se articulan como sistemas reactivos psicológicos y que tienen lugar cuando un individuo responde de cierta manera en una situación, ante personas, acontecimientos y objetos.

Kantor y Smith (1975) sostienen que un sistema reactivo es una configuración de respuestas, que se expresa como una acción integral en una situación interactiva. Los sistemas 
reactivos psicológicos articulan componentes, tanto efectivos como inefectivos (Kantor, 1924/1985) posibilitados en los humanos, por las características del medio de contacto convencional (Ribes \& Pérez Almonacid, 2012) conforme a las contingencias prevalecientes en una situación interactiva.

En el caso humano, los sistemas reactivos psicológicos están conformados predominantemente por respuestas de naturaleza lingüística; éstas ocurren en distintos modos que, dada su morfología arbitraria, se adquieren inicialmente como tres modos complementarios reactivos y activos: observar-gesticular/señalar, escuchar-hablar, y leer-escribir. Sin embargo, la adquisición de los modos reactivos antecede a la de los activos y es necesaria para retroalimentar la precisión y eficacia de los modos activos (Fuentes \& Ribes, 2001; Gómez Fuentes \& Ribes, 2008).

Los conceptos de sistema reactivo y modo de respuesta pertenecen a la lógica de las categorías modales, delimitan lo que es posible hacer aún cuando todavía no se tenga la habilidad o competencia específica (Ryle, 1949). Los modos reactivos representan funciones del individuo como lector, escucha y observador. Los activos funcionan como mediadores de otros modos, representan acciones del individuo como señalador/gesticulador, hablante y escritor. Los distintos modos lingüísticos activos y reactivos no sólo difieren respecto de los medios de ocurrencia y parámetros espacio temporales en que tienen lugar, sino también respecto de su desligabilidad situacional (Gómez Fuentes \& Ribes, 2014).

El desligamiento funcional de la respuesta respecto de las propiedades de estímulo de la situación, es uno de los cuatro conceptos básicos que aluden a distintas propiedades o características del sistema de contingencias comprendido en cada tipo de relación funcional estímulo-respuesta de la taxonomía propuesta por Ribes y López (1985). Los otros conceptos son la mediación de las relaciones de contingencia, los criterios de ajuste implicados por cada función y el tipo de relación caracterizado por la función (Ribes, 2010). Estos factores constituyen la estructura lógica de la taxonomía de funciones.

El dominio de los contenidos del conocimiento de acuerdo con Ribes (2008) se relaciona con los aspectos psicológicos que deben ser considerados en su aprendizaje: los niveles de aptitud funcional (criterios de logro en el ámbito aplicado) y habilidades que constituyen las competencias, así como los modos del lenguaje involucrados. El dominio de los contenidos del conocimiento ha estado asociado tradicionalmente al término "comprender"; su análisis ha mostrado algunos problemas al estar sustentado en un concepto ambiguo identificado como un proceso mental, de inferencia o de representación fundamentada en modelos cognitivos (Ribes, Ibáñez \& Pérez Almonacid, 2014).
Los problemas previamente señalados hacen necesaria una estrategia distinta para analizar experimental y sistemáticamente cómo ocurre la comprensión, en tanto fenómeno de aprendizaje. Ribes et al. (2014, p. 103) proponen tres criterios para su identificación y una clasificación que delimita las circunstancias de su ocurrencia.

Los criterios para identificar la comprensión se plantean a partir de su uso en el lenguaje ordinario, a saber: a) los términos comprender (verbo) y comprensión (sustantivo) pertenecen a la lógica de las categorías episódicas y de logro, respectivamente; b) el aprendizaje comprensivo es un concepto compuesto por dos términos: el primero se inserta en las categorías episódicas y de logro e implica la satisfacción de un criterio en términos de un resultado o producto; y el segundo opera como un adjetivo, que en el contexto de un episodio interactivo puede identificarse como una categoría de tipo adverbial; c) el aprendizaje comprensivo debe distinguirse de otras formas de aprendizaje por exposición reactiva en las que no opera el lenguaje como posibilitador de las interacciones.

Ribes et al. (2014) presentan una clasificación de las circunstancias en las que ocurren tipos distintos de relaciones posibles entre los modos reactivos y activos del lenguaje. El presente estudio se relaciona únicamente con una condición que el autor denomina habilitación lingüística, los otros tipos de relaciones se pueden revisar en el artículo citado.

La habilitación lingüística consiste en la facilitación del desempeño en un modo activo lingüístico (señalar, hablar, escribir) o no lingüístico (motriz) como resultado de una exposición en un modo lingüístico reactivo (observar, escuchar, leer) o no lingüístico (ver, oír). Los modos reactivos pueden ser empleados de manera aislada o combinada, dependiendo de las modalidades sensoriales utilizadas en la enseñanza: visuales, auditivas o gráficas. El efecto de dicha exposición puede evaluarse "verticalmente" en el modo activo complementario directo (señalar/gesticular para observar, hablar para escuchar, y escribir para leer), o se puede evaluar de manera "oblicua" en algunos de los modos no complementarios (hablar y escribir para observar, señalar/gesticular y escribir para escuchar, y señalar/ gesticular y hablar para leer).

Los modos reactivos no presentan modalidades de respuesta específicas, tienen un carácter orientador e implícito, a diferencia de los modos activos que tienen respuestas efectivas que satisfacen un criterio. Para evidenciar el aprendizaje de un sujeto que ha sido expuesto a un modo reactivo, se debe evaluar su ejecución ante un modo activo (Ribes, 2007a; Tamayo, Ribes \& Padilla, 2010). El proceso de aprender está ligado, de manera inseparable, a interacciones lingüísticas en las que el aprendiz despliega inicialmente y, como único requerimiento, modos reactivos 
de comportamiento lingüístico (Gómez Fuentes \& Ribes, 2008). De esta manera, el dominio de los contenidos del conocimiento o "comprensión" está vinculado con los niveles de aptitud funcional o ajuste de la ejecución bajo la modalidad activa; tal ajuste, en la habilitación lingüística, puede ser diferencial en función de la modalidad reactiva empleada al establecer contacto con los contenidos del conocimiento.

Se han realizado varias investigaciones sobre el lenguaje como comportamiento, sustentada en la teoría de la conducta. Estos estudios (Gómez Fuentes \& Ribes, 2008, 2014) han evaluado la presencia o ausencia de la retroalimentación ejercida por el modo reactivo observar, escuchar y leer ante el modo activo señalar, hablar y escribir empleando procedimientos de discriminación condicional de primer y segundo orden. Los resultados de ambos estudios mostraron que las diferencias en la adquisición, mantenimiento y transferencia de una discriminación condicional dependen del modo de respuesta lingüístico requerido al participante y de la ausencia o presencia de la retroalimentación reactiva. El análisis de la translatividad en estos estudios (efecto que tiene la adquisición de un repertorio discriminativo en un modo activo lingüístico determinado sobre su ejecución en otro modo activo lingüístico) sugirió que la ejecución de un modo a otro dependía de la facilidad en la adquisición en el modo que le precedía, las características morfológicas del modo y el grado de dificultad de la tarea.

El análisis de las relaciones entre modos activos (translatividad) condujo a Tamayo et al., (2010) a evaluar las relaciones entre modos reactivos y activos. Para identificar este tipo de relaciones los autores adoptaron el término $h a-$ bilitación y lo definieron como el conjunto de condiciones que permiten la emisión de una respuesta bajo una modalidad activa cuando el contacto con los eventos de estímulo se ha presentado bajo modalidades reactivas. En un primer estudio, Tamayo et al. (2010) analizaron la habilitación de la respuesta de igualación escrita en un procedimiento de primer orden ante la presentación de los eventos de estímulo en los modos reactivos observar, escuchar y hablar. Los resultados indicaron que el efecto de habilitación fue mayor en el modo observar en comparación con los modos leer y escuchar. Estos resultados sugirieron un segundo estudio (Tamayo \& Martínez, 2014) en el que se utilizó un procedimiento de igualación de la muestra de segundo orden, para evidenciar el reconocimiento de igualación y evaluar todas las combinaciones posibles entre modos reactivos y activos, tanto complementarios como no complementarios. $\mathrm{Al}$ igual que en el primer estudio los participantes fueron estudiantes de licenciatura. Los resultados de este estudio no corroboraron en su totalidad los hallazgos de Tamayo et al. (2010), sugiriendo que el papel dominante del modo señalar es relativo. Estos resultados pudieron verse afectados por el procedimiento de igualación empleado, de primer o segundo orden.

Por su parte Arias Pérez, Gómez Fuentes, Zepeta García \& Camacho Candía (2012) evaluaron el conocimiento aprendido durante el entrenamiento en una tarea de igualación de la muestra de segundo orden mediante los modos lingüísticos reactivos aislados o combinados, a partir del desempeño posterior en el modo activo escribir. Participaron niños de ambos sexos de cuarto grado de primaria. Los resultados sugieren que la habilitación en el modo escribir puede ser funcionalmente diferente cuando los modos reactivos se exponen de manera aislada que cuando se exponen de manera combinada y se comparte el medio de ocurrencia (i.e., auditivo o visual). Así mismo, el efecto puede ser diferente si se evalúa de manera directa (i.e., leer-escribir) o angular (i.e., observar-escribir; escuchar-escribir). Gómez Fuentes, Camacho Candia, Zepeta García, Arias Pérez \& Pérez Juárez (2012) realizaron un segundo estudio para evaluar el efecto de los modos lingüísticos reactivos en tres condiciones distintas: observar, observar-escuchar y observar-escuchar-leer, sobre la ejecución en el modo activo escribir de niños de cuarto año de primaria en una tarea de igualación de la muestra de segundo orden. En el estudio previo se identificaron problemas en el diseño de la tarea experimental, que limitaba el contacto funcional de los modos lingüísticos reactivos y activos en el episodio interactivo. Por tal motivo se realizaron algunas modificaciones a la tarea experimental: a) a las instrucciones; $y$, b) a la presentación de los estímulos: la presentación del arreglo de estímulos fue simultánea y la iluminación de los estímulos fue secuencial. Sin embargo, varios niños no identificaron el criterio de igualación. Los resultados sugieren que la utilización simultánea de los tres modos reactivos durante el entrenamiento, tiene mayor efecto en la habilitación del modo activo cuando está incluido el modo reactivo correspondiente.

El análisis planteado en el presente estudio se vincula primero, con el dominio de los contenidos del conocimiento; segundo con las interacciones lingüísticas en las que se articulan los sistemas reactivos y los modos de respuesta como categorías modales; tercero, con el aprendizaje de las relaciones de igualación como resultado de la exposición a los modos lingüísticos reactivos y cuyo efecto (habilitación) se prueba mediante los modos activos: de manera directa con el modo activo complementario o de manera "oblicua" con los modos no complementarios. 
Con base en lo anterior, el propósito del presente estudio fue evaluar el aprendizaje, resultado de la exposición al modo reactivo lingüístico observar, en los modos lingüísticos activos señalar, hablar y escribir en una tarea de igualación de la muestra de segundo orden, mediante la discriminación de relaciones de igualación por semejanza en color, y semejanza en forma, o diferencia en color y forma; dicha exposición se probó de manera "vertical" en el modo activo complementario directo: señalar, y de manera "oblicua" en los modos activos no complementarios: hablar y escribir.

\section{MÉTODO}

\section{Participantes}

Quince adolescentes experimentalmente ingenuos, doce hombres y tres mujeres, de 14 años de edad, estudiantes del tercer grado en una escuela telesecundaria pública de la ciudad de Xalapa. Los participantes se distribuyeron al azar en tres grupos experimentales de cinco integrantes cada uno. Al inicio del estudio se les dijo a los sujetos que recibirían un estímulo económico diferencial de acuerdo al número de respuestas correctas obtenidas en la prueba de habilitación; la cantidad máxima que podrían obtener era de $\$ 100.00$ pesos mexicanos.

\section{Aparatos y Situación Experimental}

Las tareas y el registro de los datos se diseñaron utilizando el programa SuperLab 4.0., bajo ambiente Windows Vista. Se realizaron cinco sesiones diarias, de 9:00 a 12:00 horas en cinco cubículos que contaban con vidrios tipo cámara de Gesell de visión unilateral, ubicados en el Laboratorio de Comportamiento Humano del Instituto de Psicología y
Educación de la Universidad Veracruzana. Cada cubículo estaba equipado con una computadora portátil Hewlett Packard tipo CP2 y audífonos.

\section{Procedimiento}

Para evaluar el efecto de los modos reactivos sobre el desempeño en los activos se utilizó un diseño experimental con tres grupos que se muestra en la tabla 1. El grupo uno (G1) se expuso a los modos observar-señalar; el grupo dos (G2) se expuso a los modos observar-hablar; y el grupo tres (G3) a los modos observar-escribir. Cada grupo se expuso a una Prueba Inicial, una Prueba de Familiarización, una Fase de Exposición y una Prueba de Habilitación.

Se empleó una tarea de igualación de la muestra de segundo orden con figuras geométricas como se muestra en la figura 1. El arreglo experimental consistió en siete estímulos presentados en la pantalla de la computadora: dos en la parte superior, indicando el criterio de igualación; un estímulo de muestra en la parte intermedia y cuatro estímulos de comparación en la parte inferior. Uno de los estímulos de comparación era idéntico al de la muestra, dos eran semejantes en forma y color, y el cuarto era diferente en forma y color. En cada sesión se presentaron ensayos de igualación por semejanza en color, semejanza en forma y de igualación por diferencia. Se presentaron 36 ensayos por sesión en la Prueba Inicial y la Prueba de Familiarización. En la Fase de Exposición y la Prueba de Habilitación se presentaron 108 ensayos, 36 ensayos por cada relación de igualación. La Prueba Inicial y la Fase de Familiarización se aplicaron en una sesión; se requirieron tres sesiones para la Fase de Exposición y una última sesión para la Prueba de Habilitación.

Tabla 1.

Diseño Experimental para analizar las relaciones funcionales entre el modo lingüístico reactivo observar y los modos activos lingüísticos señalar, hablar y escribir

\begin{tabular}{|c|c|c|c|c|c|c|}
\hline & \multicolumn{2}{|c|}{ Modos del Lenguaje } & \multirow{2}{*}{$\begin{array}{l}\text { Prueba } \\
\text { Inicial }\end{array}$} & \multicolumn{2}{|c|}{ Fases } & \multirow{2}{*}{$\begin{array}{c}\text { Prueba } \\
\text { Habilitación }\end{array}$} \\
\hline & Reactivo & Activo & & Familiarización & Exposición & \\
\hline G1 & Observar & Señalar & $\mathrm{X}$ & $\mathrm{X}$ & $\mathrm{X}$ & $X$ \\
\hline G2 & Observar & Hablar & $\mathrm{X}$ & $X$ & $\mathrm{X}$ & $\mathrm{X}$ \\
\hline G3 & Observar & Escribir & $X$ & $\mathrm{X}$ & $X$ & $X$ \\
\hline
\end{tabular}

Nota. Se evalúa verticalmente el efecto de la exposición al modo lingüístico reactivo observar en el modo lingüístico activo complementario directo señalar; de manera oblicua en los modos lingüísticos activos no complementarios hablar y escribir. 

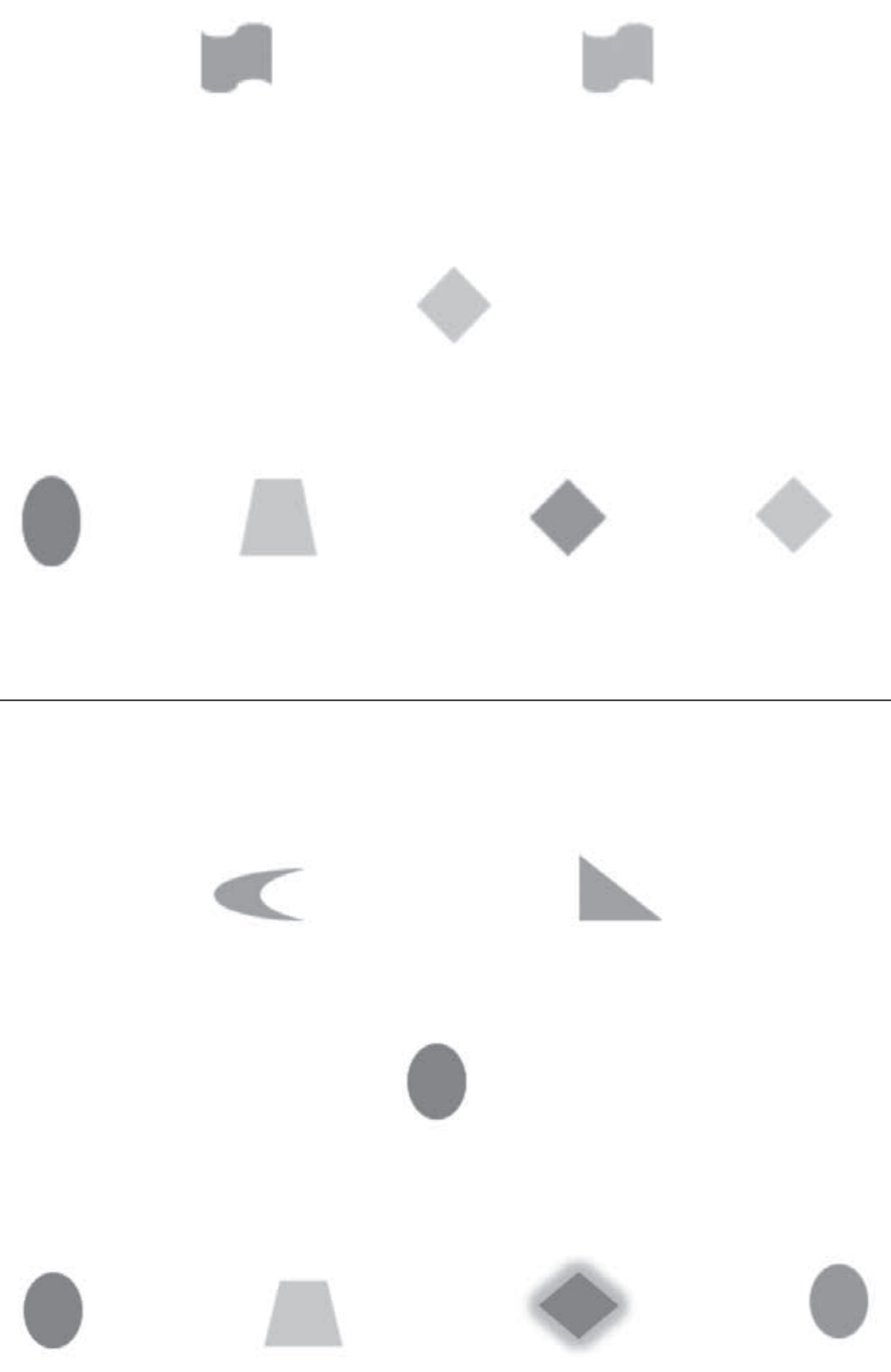

Figura 1. Habilitación Lingüística. Arreglo experimental de segundo orden. Prueba Inicial y Prueba de Habilitación (parte superior); Fase de Exposición (parte inferior). 


\section{Prueba Inicial}

Se utilizó una tarea de igualación de la muestra de segundo orden construida con el $33 \%$ de los ensayos de la Fase de Exposición (se incluyeron relaciones por semejanza en color, semejanza en forma y diferencia en color y forma). El sistema de cómputo presentaba simultáneamente los siete estímulos del arreglo experimental. Al inicio de la sesión se presentó una instrucción general en la pantalla de la computadora y los participantes realizaron una lectura silente. Después observaron-señalaron (G1), observaron-hablaron (G2) y observaron-escribieron (G3) en la computadora la forma y el color de las figuras geométricas. En esta prueba el modo de respuesta fue señalar, hablar o escribir. No se informó a los participantes si las respuestas eran correctas o incorrectas. La instrucción general en esta prueba fue la siguiente:

En la siguiente pantalla se te proporcionarán instrucciones sobre cómo resolver la tarea que vas a realizar. Para comenzar, oprime con el botón izquierdo del mouse INICIAR.

En las siguientes pantallas aparecerán siete figuras geométricas: dos en la parte superior, una en el centro y cuatro en la parte inferior. De las figuras de abajo, elige aquella que corresponde con la del centro de acuerdo con lo que indican las dos figuras de arriba. Para registrar tu respuesta ubica el puntero del mouse dentro de la figura que elegiste y oprime el botón izquierdo. (Para los otros modos de respuesta se realizaron las modificaciones pertinentes).

\section{Fase de Familiarización}

Las instrucciones generales y específicas fueron similares a las utilizadas durante la Prueba Inicial. Esta fase se realizó para favorecer el contacto funcional con la tarea experimental y el uso del equipo de cómputo. Se expuso a los participantes a una tarea en el modo reactivo observar y se evaluó en distintos modos activos de respuesta empleando un arreglo experimental de primer orden. De esta manera se podría suponer que las respuestas emitidas en la Prueba de Habilitación se debían a la identificación del criterio de igualación. No se informó a los participantes si las respuestas emitidas eran correctas o incorrectas.

\section{Fase de Exposición}

Los participantes de los tres Grupos fueron expuestos a tareas de segundo orden, empleando el modo observar. Las instrucciones fueron similares a las de la Prueba Inicial. El sistema de cómputo presentaba simultáneamente los siete estímulos del arreglo experimental; después se iluminaba el contorno de los estímulos geométricos uno después de otro. La tarea de los participantes en los tres grupos consistía en identificar la relación por semejanza (en color o forma) o diferencia (en color y forma) del estímulo de comparación con el estímulo de muestra de acuerdo con la relación indicada por los estímulos de segundo orden. El sistema de cómputo emitía la respuesta correcta iluminando el estímulo de comparación correcto. Además, se incluía una "palomita" verde para indicar que la relación con el estímulo de muestra era correcta. El participante no emitía ninguna respuesta.

\section{Prueba de Habilitación}

Esta prueba fue igual a la prueba inicial, se utilizó para evaluar el efecto del modo reactivo observar en la identificación de las relaciones de semejanza (color o forma) y diferencia (color y forma) empleando los modos activos señalar, hablar y escribir. En el modo señalar, el participante emitía la respuesta dando un clic en la figura que consideraba correcta; en el segundo modo, ante la imagen de un micrófono el participante decía el nombre y el color de la figura que consideraba correcta. Para el modo escribir, después de presentar el arreglo experimental, aparecía otra pantalla con un cuadro de texto, en la que el participante escribía el nombre y color de la figura que consideraba correcta. Las instrucciones fueron similares a las de la Prueba Inicial. No se informó a los participantes si las respuestas eran correctas o incorrectas.

\section{RESULTADOS}

La figura 2 muestra el efecto "vertical" de la exposición al modo reactivo lingüístico observar en el modo lingüístico activo complementario directo señalar y el efecto de manera "oblicua" de la exposición al mismo modo reactivo en los modos lingüísticos activos no complementarios hablar y escribir. En la parte superior, se muestran altos porcentajes de respuestas correctas en el modo activo señalar en dos participantes: P01 96\% y P05 99\%; el P04 alcanzó el 52\% de aciertos y los dos participantes restantes obtuvieron porcentajes menores al 34\%. En la parte media de la figura 2, se observan porcentajes altos en los cinco participantes: P06 94\%, P07 96, P08 95\%, P09 96\% y P010 95\%. En la parte inferior de la misma figura, los resultados muestran porcentajes altos en el modo activo escribir en cuatro participantes: P11 91\%, P13 92\%, P14 90\% y P15 98\%. El P012 mostró un porcentaje menor al 14\% de aciertos.

La figura 3 describe, en porcentajes, el total de respuestas correctas $(100 \%)$ emitidas por cada participante, distribuido en los tres tipos de relación de igualación (semejanzas en color y forma y diferencia) evaluadas en las Pruebas Inicial y de Habilitación. La figura 3, parte inferior y columna de la izquierda, muestra que en la Prueba de Habilitación los participantes $\mathrm{P} 01$ y P05, que alcanzaron altos porcentajes 


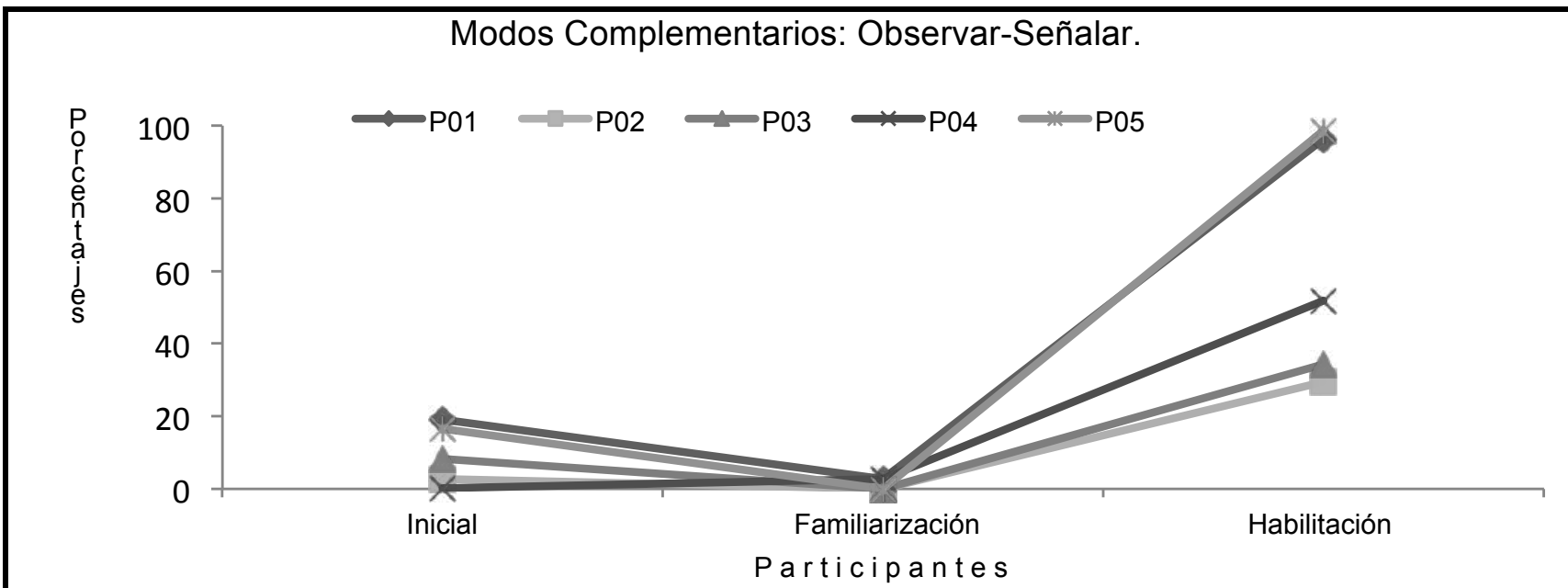

Modos no Complementarios: Observar-Hablar.

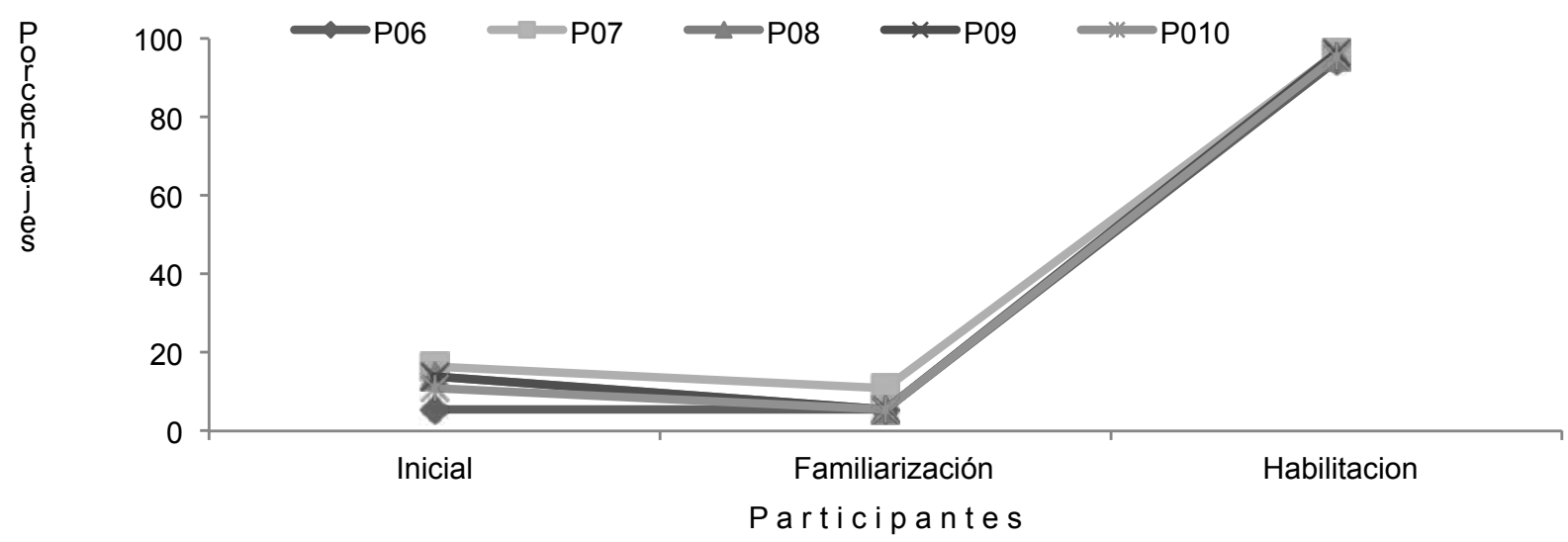

Modos no Complementarios: Observar-Escribir.

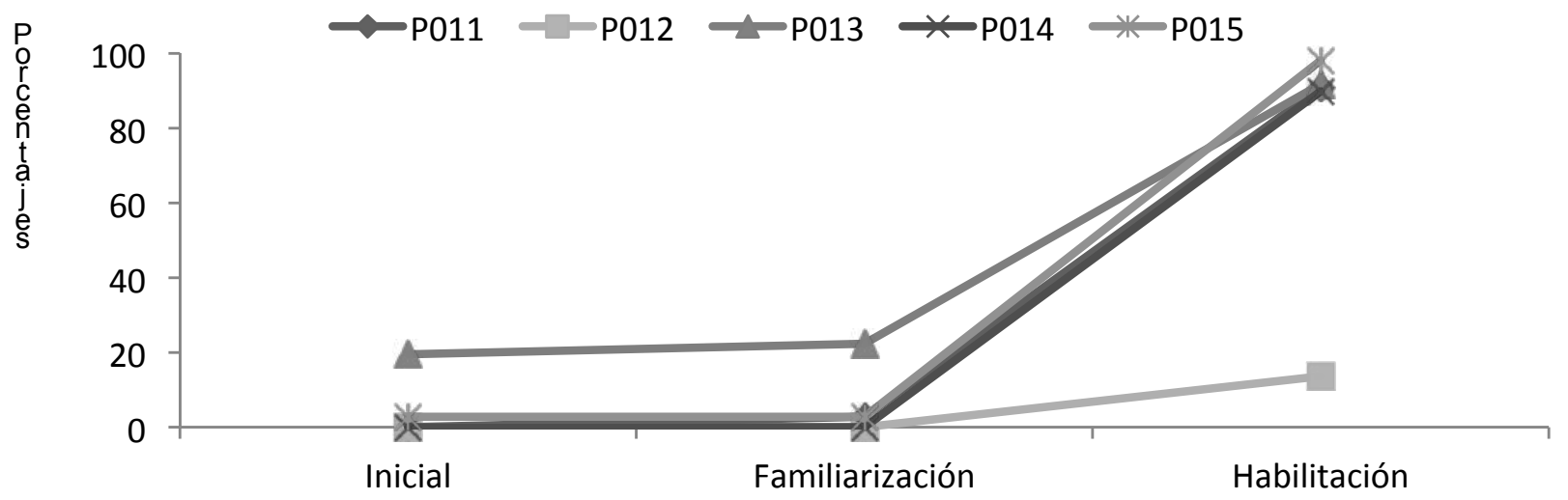

Participantes

Figura 2. Habilitación Lingüística vertical (parte superior) y oblicua (parte central e inferior). Porcentaje de respuestas correctas durante las Pruebas Inicial, Habilitación y Fase de Familiarización. 


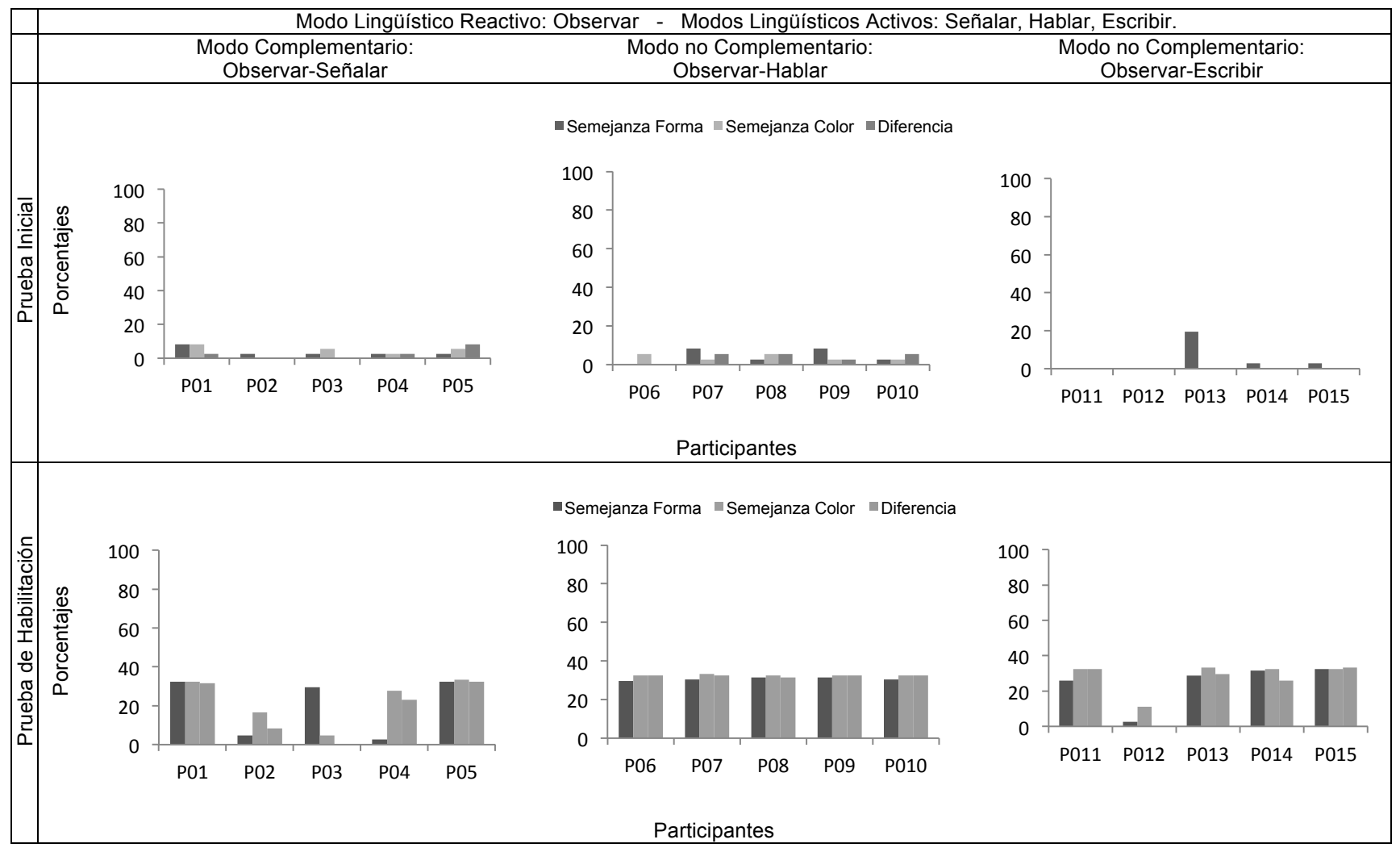

Figura 3. Habilitación Lingüística. Relaciones de igualación por semejanza en forma y color, y por diferencia. Porcentaje de respuestas correctas: Modo lingüístico reactivo observar y Modo lingüístico activo complementario directo señalar; Modo lingüístico reactivo observar y Modos lingüísticos activos no complementarios hablar y escribir.

de respuestas correctas en el modo activo señalar, tuvieron ejecuciones similares en los tres tipos de igualación, aunque P05 mostró un porcentaje ligeramente mayor en la relación por semejanza en color. En la parte inferior y columna central, los resultados indican que los cinco participantes alcanzaron porcentajes altos de ejecución; estos porcentajes se distribuyeron de manera similar en las tres relaciones de igualación, aunque en promedio un poco mayor en la relación por semejanza en color (33\%); en las relaciones por diferencia y semejanza en forma los participantes mostraron, respectivamente, los siguientes porcentajes promedio: $32 \%$ y $31 \%$. Finalmente, en la parte inferior, columna de la derecha, los cuatro participantes con altos niveles de desempeño, mostraron ejecuciones similares en los tres tipos de igualación, aunque un poco mayor en semejanza en color 33\%, seguido de diferencia y semejanza por forma con un $30 \%$.

\section{DISCUSIÓN}

Los resultados muestran que la exposición de los participantes al modo lingüístico observar en la Fase de Exposición, utilizando una tarea de igualación de la muestra de segundo orden en la que se emplearon relaciones de igualación por semejanza en forma, color y diferencia, facilitó el desempeño en los modos activos lingüísticos en la Prueba de Habilitación: mayor en hablar, luego en escribir y menor en señalar. Los participantes con desempeños altos en los tres modos activos mostraron niveles de ejecución similares en los tres tipos de igualación, aunque un poco mayor en semejanza en color, luego en diferencia y menor en semejanza en forma.

Así, se observan efectos de habilitación, distintos en los tres modos activos. En señalar, modo complementario directo, sólo dos participantes alcanzaron porcentajes mayores al 90\%; sin embargo, los porcentajes obtenidos individualmente fueron los más altos. En cambio, en hablar y escribir, modos no complementarios, este porcentaje fue obtenido por cinco y cuatro participantes respectivamente, aunque en promedio los porcentajes fueron mayores que en el modo señalar. Estos hallazgos fueron parcialmente distintos a los de Tamayo y Martínez (2014) quienes reportaron que el modo observar habilitó en mayor grado la respuesta de igualación en los modos escribir y señalar; en estos modos, tres participantes obtuvieron porcentajes de aciertos mayores al 90\% en la Prueba de Habilitación, 
y en el modo hablar sólo dos participantes alcanzaron este porcentaje.

Tamayo et al. (2010) reportaron que el modo observar habilitó en mayor grado la respuesta de igualación escrita en comparación con las modalidades leer y escuchar. A partir de estos hallazgos, sugirieron que el modo observar puede tener un papel funcional central en las modalidades lingüísticas reactivas de la misma forma que el modo hablar presenta tal característica en los modos activos (Gómez Fuentes \& Ribes, 2008). Sin embargo, Tamayo y Martínez (2014), sugirieron que el efecto nodal del modo observar con respecto a los modos reactivos escuchar y leer es relativo.

En los estudios de Tamayo et al. (2010) y, Tamayo y Martínez (2014), se evaluó el efecto de la habilitación lingüística en los modos activos lingüísticos señalar, hablar y escribir. Sin embargo, no se consideró el efecto que pudiera tener la exposición aislada o combinada a los modos lingüísticos reactivos. Cada tipo de exposición reactiva puede tener efectos de habilitación distintos. Arias et al. (2012) y Gómez Fuentes et al. (2012) evaluaron la exposición de los modos lingüísticos reactivos de manera aislada y combinada, y probaron su efecto en el modo lingüístico escribir. Los resultados de ambos estudios sugieren que la utilización simultánea de los tres modos reactivos durante la Fase de Exposición puede tener mayor efecto en la habilitación del modo activo cuando está incluido el modo reactivo complementario.

Los resultados de los estudios previamente citados sugieren que las variables que regulan un modo lingüístico no son funcionales en los otros modos; esto se puede deber al medio de ocurrencia (i.e. visual, auditivo) de cada uno de los modos lingüísticos, y a los parámetros espacio temporales que posibilitan interacciones de distinto nivel funcional (Gómez Fuentes \& Ribes, 2014).

Los resultados descritos en el presente estudio muestran niveles de ejecución similares en la discriminación de las relaciones de semejanza en forma, semejanza en color, y diferencia en color y forma en los participantes que alcanzaron altos porcentajes de respuestas correctas en la Prueba de Habilitación, independientemente de las características morfológicas y funcionales de los modos lingüístico activos. En este estudio, la exposición a un mismo modo reactivo podría ser un factor relevante en el nivel de ejecución mostrado en los modos activos. También se observó que la discriminación de las relaciones de igualación por semejanza en color fue un poco mayor, seguida por la igualación por diferencia y menor por forma. Estos resultados sugieren que el "ver" como modalidad no lingüística (Ribes et al., 2014) puede favorecer o limitar el efecto de habilitación. El análisis de los hallazgos reportados por Tamayo et al. (2010, 2014), y los del presente estudio sugieren que la exposición a los modos reactivos facilita el desempeño en la discriminación de las relaciones de igualación en la Prueba de Habilitación, aunque el efecto en cada modo activo del lenguaje puede ser distinto; esto puede depender del modo reactivo utilizado en la Fase de Exposición.

Los resultados observados en los tres modos activos en la Fase de Familiarización (figura 2), muestran porcentajes menores a los obtenidos en la Prueba Inicial. Es decir, los participantes no discriminaron el criterio de igualación (semejanza en forma o color, y diferencia) implicado en la tarea experimental de primer orden. Esto puede deberse a que el medio de contacto convencional no posibilitó, en la tarea experimental, la interacción entre los acontecimientos o eventos constituidos por las acciones o reacciones del organismo y los objetos de estímulo. Una característica distintiva del comportamiento humano, señala Ribes (2007b) es la naturaleza convencional de sus sistemas reactivos dominantes, articulados a partir y como lenguaje.

El bajo desempeño se puede deber también a los factores de procedimiento implicados en el arreglo experimental de primer orden. En este estudio no se informó a los participantes si las respuestas emitidas eran correctas o incorrectas. En tareas de primer orden, en ausencia de instrucciones explícitas, la única forma en la que el participante puede discriminar el criterio de igualación (semejanza en forma o color, y diferencia) prevaleciente es por medio de la retroalimentación de sus respuestas. De acuerdo con Ribes y Martínez (1990), y Ribes y Torres (2001), la retroalimentación es fundamental para discriminar el criterio de igualación.

Los hallazgos del presente estudio y los reportados por Tamayo et al. $(2010,2014)$ sugieren que el efecto de la exposición al modo observar en los modos activos es distinto, y que su efecto nodal es relativo. El efecto de habilitación puede estar relacionado con los siguientes aspectos: primero, con las características funcionales y morfológicas de los modos reactivos y activos del lenguaje (Gómez Fuentes \& Ribes, 2008); segundo, con el tipo de relación entre modos reactivos (lingüísticos y no lingüísticos) y activos (lingüísticos y no lingüísticos): a) exposición aislada o combinada de los modos reactivos; b) con el tipo de evaluación de los modos activos: "vertical" en el modo activo complementario directo o "oblicuo" en los modos activos no complementarios (Ribes et al. 2014); tercero, con la relación nodal que el modo activo hablar (Gómez Fuentes \& Ribes, 2008, 2014), y el modo reactivo observar (Tamayo \& Martínez, 2014) pueden tener con los otros modos lingüísticos durante la adquisición, mantenimiento y transferencia del comportamiento; cuarto, con el arreglo experimental utilizado, de primer o segundo orden (Gómez Fuentes \& Ribes, 2008, 2014; Tamayo \& Martínez, 2014; Tamayo et al. 2010), el diseño de las tareas y otras con- 
diciones que se introducen en el procedimiento (Arias et al. 2012; Gómez Fuentes et al., 2012), por ejemplo, pagar a los sujetos experimentales por participar en el estudio, la presentación simultánea o secuencial de los estímulos geométricos y sus características sensoriales. Los estudios mencionados sugieren que es importante identificar las relaciones posibles entre los distintos modos lingüísticos reactivos y activos; su estudio sistemático puede contribuir al estudio de la habilitación.

Varios son los problemas que se deben enfrentar para la operación de los planes y programas de estudio propuestos en la Reforma de la Educación Básica (SEP, 2011a), unos tecnológicos y otros teóricos. Respecto a los primeros, no es suficiente señalar que la reforma se organiza en campos formativos y dominios de conocimiento con fundamento en el concepto de competencia como capacidad general, sino que es necesario cambiar la lógica tradicional centrada en el que enseña. Ribes ya ha planteado una propuesta centrada en el aprendiz, en el ámbito de la educación básica (Ribes, 2008; Ribes et al. 2014). En este contexto, como una extensión de la Teoría de la Conducta (Ribes \& López, 1985), el término competencia puede constituirse en un instrumento útil par la formación académica y práctica de los estudiantes. (Ribes, 2006, 2008). Para ello es necesario abordar el aprendizaje de los contenidos del conocimiento como un problema psicológico contextualizado en situaciones específicas (i.e. niveles educativos, campos formativos) con diferentes criterios de logro.

El problema teórico, vinculado con la identificación de las posibles interacciones entre los modos reactivos y activos del lenguaje en el dominio o "comprensión" de los contenidos del conocimiento es relevante, no sólo en la investigación básica sino también en la aplicada. En el episodio interactivo participan los modos lingüísticos reactivos y activos. En este contexto, una línea de investigación podría estar dirigida a analizar los factores que probabilizan una interacción sin participar directamente en ella: el contacto reactivo tendría que especificar un efecto facilitador de naturaleza histórica en la etapa de exposición a los modos reactivos. De acuerdo con Ribes et al. (2014), hay dos formas posibles. En la primera, la etapa activa debe contener contingencias situacionales propias de la etapa reactiva; en la segunda, la etapa activa debe contener contingencias situacionales distintas a las de la etapa reactiva. En esta línea de investigación se podría evaluar la exposición a los modos reactivos de forma aislada o simultánea, dependiendo de las modalidades sensoriales utilizadas en la enseñanza. Otra línea de investigación, abordada parcialmente en el presente estudio, sería evaluar el efecto de habilitación en los modos lingüísticos activos de manera "vertical" en el modo activo complementario o de manera "oblicua" en los modos activos no complementarios. Así, se pueden evaluar también las interacciones entre los modos reactivos y activos del lenguaje en situaciones nuevas no entrenadas de manera directa. Es decir, en un mismo nivel de aptitud, o en distinto nivel, empleando pruebas de transferencia (e.g. intramodal, extramodal, extrarelacional).

Otra línea importante de investigación, orientada al estudio de los modos lingüísticos, que pudiera ser explorada está vinculada con la adquisición de habilidades y aptitudes y, que constituyen las competencias. Desde la perspectiva de la Teoría de la Conducta (Ribes \& López, 1985), las competencias se identifican con base en criterios funcionales de logro explícitos, es decir, como actos lingüísticos en una situación determinada. Este análisis se puede realizar desde tres perspectivas distintas: primero, mediante el efecto de habilitación en los modos activos; segundo, como componentes que facilitan la adquisición de una competencia; y tercero, como facilitadores de desempeños efectivos en distintos niveles de aptitud funcional (Ribes et al., 2014). En conclusión, la habilitación como un tipo de comprensión, es un concepto de tipo disposicional de carácter episódico ligado a interacciones lingüísticas en las que participan los modos reactivos y activos del lenguaje.

\section{REFERENCIAS}

Arias Pérez, S., Gómez Fuentes, A. D., Zepeta García, E., \& Camacho Candia, J. A. (2012). Análisis del conocimiento aprendido en los modos reactivos y su efecto en el modo escribir. En A. D. Gómez Fuentes, G. Reyes Hernández, M. W. Salas Martínez, \& E. Zepeta García, Investigación en Psicología Aplicada a la Educación. Tomo IV. (pp. 34-52). Xalapa, Veracruz: Universidad Veracruzana-IETEC.

Fuentes, M., \& Ribes, E. (2001). Un análisis funcional de la comprensión lectora como interacción conductual. Revista Latina de Pensamiento y Lenguaje, 9(2), 181-212.

Gómez Fuentes, A. D., Camacho Candia, J. A., Zepeta García, E., Arias Pérez, S., \& Pérez Juárez, M. (2012). Entrenamiento en los modos reactivos y su efecto en el modo activo escribir. IPyE: Psicología y Educación, 6(12), 28-44.

Gómez Fuentes, A. D., \& Ribes E. (2008). Adquisición y transferencia de una discriminación condicional del primer orden en distintos modos del lenguaje. Acta Comportamentalia, 16(2), 183-209.

Gómez Fuentes, A. D., \& Ribes Iñesta, E. (2014). Adquisición y transferencia de una discriminación condicional de segundo orden en distintos modos de lenguaje. Journal of Behavior Health \& Social Issues, 6(1), 89-106.

Kantor, J. R. (1924/1985). Principles of psychology. Chicago: The Principia Press.

Kantor, J. R., \& Smith, W. (1975). The science of psychology: an interbehavioral survey. Chicago: The Principia Press. 
Ribes, E. (2006). Competencias conductuales: su pertinencia en la formación y práctica profesional del psicólogo. Revista Mexicana de Psicología, 23, 19-26.

Ribes, E. (2007a). Lenguaje, aprendizaje y conocimiento. Revista Mexicana de Psicología, 24(1), 7-14.

Ribes, E. (2007b). Estados y límites del campo, medios de contacto y análisis molar del comportamiento: reflexiones teóricas. Acta Comportamentalia, 15(2), 229-259.

Ribes, E. (2008). Educación básica, desarrollo psicológico y planeación de competencias. Revista Mexicana de Psicología, 25(2), 193-207.

Ribes, E. (2010). Teoría de la conducta 2. Avances y extensiones. México: Trillas.

Ribes, E. (2011). El concepto de competencia: su pertinencia en el desarrollo psicológico y la educación. Bodón, 63(1), 33-45.

Ribes, E., Ibáñez, C., \& Pérez Almonacid, R. (2014). Una propuesta metodológica para el análisis experimental del aprendizaje comprensivo. Revista Mexicana de Psicología, 31(1), 100-110.

Ribes, E., \& López, F. (1985). Teoría de la conducta. Un análisis de campo y paramétrico. México: Trillas.

Ribes, E., \& Martínez, H. (1990). Interaction of contingencies and rule instructions in the performance of human subjects in conditional discrimination. The Psychological Record, 40, 565-586.
Ribes, E., \& Pérez Almonacid, R. (2012). La función lógica del concepto de medio de contacto. Acta Comportamentalia, 20(2), 235-249.

Ribes, E., \& Torres, C. J. (2001). Un estudio comparativo de los entrenamientos de primer y segundo orden en igualación de la muestra. Revista Mexicana de Análisis de la Conducta, 27, 385-401.

Ryle, G. (1949). The concept of mind. London: Routledge.

Secretaría de Educación Pública. (2011a). Plan de estudios 2011. Educación Básica. México: Autor.

Secretaría de Educación Pública. (2011b). Reforma Integral, diplomado para maestros, $3^{\circ}$ y $4^{\circ}$ grado modulo 1 , fundamentos de la educación básica. México: Autor.

Secretaría de Educación Pública. (2011c). Acuerdo Número 592, por el que se establece la articulación de la Educación Básica. México: Autor.

Tamayo, J., Martínez, D. R. (2014). Efecto de habilitación en un procedimiento de igualación de la muestra de segundo orden. Revista Mexicana de Investigación en Psicología, 6(1), 97-108.

Tamayo, J., Ribes, E., \& Padilla Vargas, M. A. (2010). Análisis de la escritura como modalidad lingüística. Acta Comportamentalia, 18(1), 87-106. 